\title{
Catalogue of Syringogastridae (Diptera, Diopsoidea)
}

\author{
Alessandre Pereira-Colavite ${ }^{1}$ \& Ramon Luciano Mello ${ }^{2}$ \\ 1 Universidade Federal da Paraíba (UFPB), Centro de Ciências Exatas e da Natureza (CCEN), Departamento de Sistemática e Ecologia (DSE). \\ João Pessoa, PB, Brasil. ORCID: 0000-0002-7660-8384. E-mail: alepercol@gmail.com \\ ${ }^{2}$ Universidade Federal de Mato Grosso do Sul (UFMS), Instituto de Biociências (INBI0), Coordenaç̧ão de Gestão Acadêmica (COAC). \\ Campo Grande, MS, Brasil. ORCID: 0000-0002-1914-5766. E-mail: ramon.mello@ufms.br
}

\begin{abstract}
The catalogue of the Syringogastridae is updated, including now 21 extant species and two fossil records, all belonging to the genus Syringogaster Cresson. References to all known bibliography are given, totaling 27 records. A full list of the type-series and distribution records are also presented.
\end{abstract}

Key-Words. List of species; Neotropical region; Taxonomy; Type material.

\section{INTRODUCTION}

The Syringogastridae Prado comprises only a single genus of ant-like flies, Syringogaster Cresson (Fig. 1), with 21 described extant species and two fossils. Syringogastrids occur throughout tropical areas of the Neotropical Region - with exception of the Antilles, from which they are known only by the fossil - reaching from borders of neotropics in Mexico (S. subnearctica Feijen) south to Misiones, Argentina (an unassociated female similar to S. carioca Prado). The species can be found in lowland tropical forests from sea level to medium elevations $(800 \mathrm{~m})$, but occasionally they may be found up to 1,450 m (Marshall et al., 2009).

Syringogastrids flies can be recognized by the petiolate abdomen, the long and collar-like prothorax, a swollen and spinose hind femur, and reduced head chaetotaxy (Marshall et al., 2009; Marshall \& Buck, 2010) (Fig. 1). The biology is basically unknown. Some species were observed in presumably mating aggregations on the upper surfaces of broadleaved foliage and feeding in extrafloral nectaries - females observed had swollen abdomens, possibly replete with honeydew (Marshall et al., 2009). Papavero (1964) described syringogastrids on leaves walking and moving similar to ants of the genus Pseudomyrmex Lund occurring on the same leaf surfaces, and Marshall \& Buck (2010) noticed syringogastrids occuring in foliages with Globopeza Marshall and Metasphen Frey (Micropezidae), all three myrmecomimicring. No elaborated courtship is known occurring in the family. Feijen (1989) and Meier \& Hilger (2000) provided some information about egg morphology, but larva and pupa still remain unknown.
The systematics of the family follows: Cresson (1912) erected Syringogaster to two species and placed the genus within the Psilidae. Curran (1934) classified Syringogaster tentatively in the Megamerinidae, an opinion shared by Brues \& Melander (1932), Brues et al. (1954) and Hennig (1958). Prado (1969), based in the principles of the phylogenetic systematics, erected the family Syringogastridae for Syringogaster and described six species from Brazil, a view shared by later authors (Griffiths, 1972; Hennig, 1973; J. McAlpine, 1989; D. McAlpine, 1997). Feijen (1983, 1989) described one species and placed Syringogastridae as sister-group of Centrioncidae (sensu Feijen), but this viewpoint was strongly rejected based in morphological and molecular data (D. McAlpine, 1997; Meier \& Hilger, 2000) in truth, Syringogastridae is the sister-group of Diopsidae [Diopsinae + Centrioncinae] (Marshall, 2012). Marshall et al. (2009) reviewed the entirely family under a systematic approach and added 11 extant species and two fossils. Rafael et al. (2011) added one extant species more.

The family is currently classified in groups and subgroups according the phylogenetic relationships (Marshall et al., 2009; Rafael et al., 2011): brunnea-group, with brunnea-subgroup [S. brunnea Cresson, S. carioca Prado and S. subnearctica Feijen], brunneina-subgroup [S. brunneina Marshall \& Buck, S. cressoni Prado, S. lopesi Prado, S. palenque Marshall \& Buck and S. sharkeyi Marshall \& Buck] and S. nigrithorax Marshall \& Buck; the fossil craigi-group [S. craigi Gaimari and S. miocenecus Gaimari]; figurata-group, with figurata-subgroup [S. atricalyx Marshall \& Buck, S. figurata Marshall \& Buck, S. plesioterga Marshall \& Buck and certainly S. amazonensis Prado] and S. dacty- 

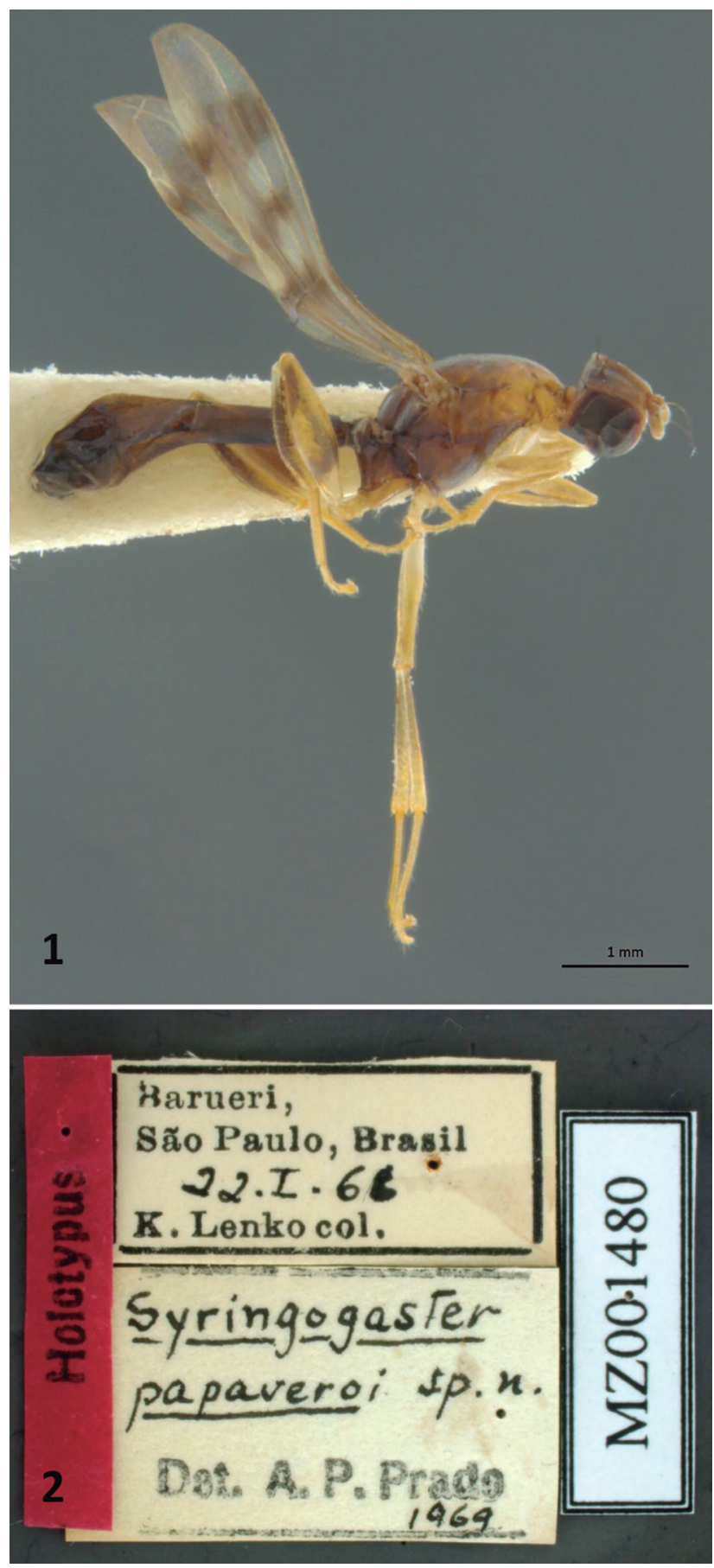

Figures 1-2. Syringogaster papaveroi Prado, holotype: (1) habitus; (2) labels.

lopleura Marshall \& Buck; rufa-group, with lanei-subgroup [S. lanei Prado and S. papaveroi Prado] and rufa-subgroup [S. apiculata Marshall \& Buck, S. brachypecta Marshall \& Buck, S. rufa Cresson and S. tenuipes Marshall \& Buck] and S. fapeam Rafael, Câmara \& Holanda.

This catalogue updates the previously of Prado (1975, 8 spp.) including now 23 species names in total, till the deadline of August $1^{\text {st }}$ of 2017.

\section{MATERIAL AND METHODS}

The abbreviations and symbols used in the text follow: cat. (catalogue), char. (characterization); desc. (de- scription), fig./figs. (figure/figures), ICZN (International Code of Zoological Nomenclature), phyl. (phylogeny), pl./pls. (plate/plates), orig. des. (by original designation), redesc. (redescription), Refs. (references), † (fossil species). The type-series is listed in full. Taxonomic and systematics literature are given. Distribution records are listed by country, in alphabetical order, with respective provinces, departments and states in parentheses.

The acronyms of the museums and collections cited are: AMNH - American Museum of Natural History, New York, New York, USA; ANSP - Academy of Natural Sciences, Philadelphia, Pennsylvania, USA; CAS - California Academy of Sciences, San Francisco, California, USA; CBFC - Colección Boliviana de Fauna, La Paz, Bolivia; CNCI - Canadian National Collection of Insects and Arachnids, Ottawa, Ontario, Canada; CSCA - California State Collection of Arthropods, Sacramento, California, USA; DEBU - University of Guelph Insect Collection, Guelph, Ontario, Canada; FIOC - Fundação Instituto Oswaldo Cruz, Rio de Janeiro, Brazil; INBC - Instituto Nacional de Biodiversidad, Santo Domingo de Heredia, Costa Rica; INPA - Instituto Nacional de Pesquisas da Amazônia, Manaus, Brazil; LACM - Natural History Museum of Los Angeles County, Los Angeles, California, USA; MUSM Museo de Historia Natural, Universidad Nacional Mayor de San Marcos, Lima, Peru; MZUSP - Museu de Zoologia da Universidade de São Paulo, São Paulo, Brazil; QCAZ Universidad Católica del Ecuador, Quito, Ecuador; ROME - Royal Ontario Museum, Toronto, Ontario, Canada; SMTD - Staatliches Museum für Tierkunde, Dresden, Germany; UASC - Museo de Historia Natural Noel Kempff Mercado, Santa Cruz, Bolivia; USNM - National Museum of Natural History, Washington D.C., USA; UWIC - Insect Collection of the Department of Biological Sciences, University of the West Indies, St. Augustine, Trinidad.

This catalogue follows the alphabetic order to species and references to groups and subgroups are listed after them.

\section{Catalogue}

\section{Syringogastridae Prado, 1969}

Syringogastridae Prado, 1969: 4 (desc.), 23 (taxonomy, relationships), 31 (phylogenetic scheme). Typegenus: Syringogaster Cresson, 1912. Refs. - Griffiths, 1972: 168-169 (systematics), 268 (key); Hennig, 1973: 15 (key), 53 (diagnosis); Prado, 1975: 1-2 (cat.); Feijen, 1983: 3-137 (systematics, sister-group of Centrioncidae sensu Feijen); Feijen, 1989: 1-122 (systematics, char.); J. McAlpine, 1989: 1431 (fig. 116.2, cladogram), 1432-1436 (systematics); D. McAlpine, 1997: 167-194 (systematics), 184 (key); Meier \& Hilger, 2000: 1-36 (systematics, sister-group of Diopsidae); Prado \& Papavero, 2002: 1 (cat., Amazon); Marshall et al., 2009: 1-80 (review); Buck et al., 2009: 137 (key); Marshall \& Buck, 2010: 843-846 (manual); Marshall, 2012: 346-348 (char.), 590 (key); Rung, 2016: 558-560 (cat., Colombia). 


\section{Syringogaster Cresson, 1912}

Syringogaster Cresson, 1912: 392. Type-species: S. rufa Cresson, 1912 (orig. des.). Refs. - Cresson, 1912: 392 (desc., in Psilidae); Brues \& Melander, 1932: 328 (fig.), 329 (key, in Megamerinidae); Curran, 1934: 26 (key), 373 (in Megamerinidae; figs.); Brues et al., 1954: 368 (fig.), 369 (key, in Megamerinidae); Hennig 1958: 568 (systematics, in Megamerinidae); Hennig, 1965: 51-52 (notes); Roback, 1969: 522 (cat.); Prado, 1975: 1 (cat.); D. McAlpine 1997: 190-194 (figs.); Marshall et al., 2009: 15 (redesc.); D. McAlpine, 2011: 148-149 (figs.); Rung, 2016: 558-560 (cat.).

amazonensis Prado, 1969: 12. Type locality: Brazil, Pará, Estrada Belém-Bragança, km 100. Distr. - Brazil (Pará). Holotype,+ FIOC. Refs. - Prado, 1969: 6 (key), 9 (fig.), 12 (desc.), 13 (fig.); Prado, 1975: 1 (cat.); Prado \& Papavero, 2002: 1 (cat.); Marshall et al., 2009: 18 (key, notes), 19 (distr. map).

apiculata Marshall \& Buck, 2009: 20 (in Marshall et al., 2009). Type locality: Ecuador, Departamento Orellana, near Yasuní National Park, Tiputini Biodiversity Station, 00³7'55"S, 7608'39"W, 220-250 m. Distr. Brazil (Amazonas), Ecuador (Napo, Orellana), Peru (Madre de Díos). Holotype ơ, USNM. Refs. - Marshall et al., 2009: 18 (key), 19 (distr. map), 20 (desc.), 21 (figs.), 58 (pl.); Rafael et al., 2011: 32 (new records), 33 (fig., phyl.).

atricalyx Marshall \& Buck, 2009: 22 (in Marshall et al., 2009). Type locality: Bolivia, La Paz, Heath River Wildlife Centre, $12^{\circ} 40^{\prime} \mathrm{S}, 68^{\circ} 42^{\prime} \mathrm{W}$. Distr. - Bolivia (Cochabamba, La Paz), Brazil (Acre, Amazonas, Pará), Ecuador (Napo, Orellana, Pastaza, Sucumbíos), Peru (Huánuco, Loreto, Madre de Díos). Holotype ơ, UASC. Refs. - Marshall et al., 2009: 13 (fig., phyl.), 18 (key), 19 (distr. map), 22 (desc.), 23 (figs.), 34 (pl.), 58 (pl.), 67-68 (pls.); Rafael et al., 2011: 32 (new records), 33 (fig., phyl.); Marshall, 2012: 475 (fig.).

brachypecta Marshall \& Buck, 2009: 25 (in Marshall et al., 2009). Type locality: Ecuador Napo, Jatun Sacha Reserve, $6 \mathrm{~km}$ E Misahuallí, $450 \mathrm{~m}, 01^{\circ} 04^{\prime} \mathrm{S}, 77^{\circ} 37^{\prime} \mathrm{W}$. Distr. - Ecuador (Napo, Orellana, Sucumbíos), Peru (Madre de Díos). Holotype o", QCAZ. Refs. - Marshall et al., 2009: 18 (key), 19 (distr. map), 25 (desc.), 26 (figs.), 58 (pl.), 67-68 (pls.); Rafael et al., 2011: 33 (fig., phyl.).

brunnea Cresson, 1912: 394. Type locality: Costa Rica, Peralta Station. Distr. - Colombia (Valle del Cauca), Costa Rica (Cartago, Heredia, Puntarenas), Ecuador (Guayas), Guatemala (Izabal), Panama (Panamá, Coclé). Holotype ${ }^{\prime}$, ANSP. Refs. - Cresson, 1912: 394 (desc.); Roback, 1969: 530 (cat., type material); Prado, 1969: 22 (notes); Prado, 1975: 1 (cat.); Buck et al., 2009: 128 (fig.); Marshall et al., 2009: 13 (fig., phyl.), 17 (key), 19 (distr. map), 28 (redesc.), 29 (figs.), 34 (pl.), 45 (pl.); Marshall \& Buck, 2010: 843 (fig.); Rafael et al., 2011: 33 (fig., phyl.); Rung, 2016: 559 (cat.).

brunneina Marshall \& Buck, 2009: 30 (in Marshall et al., 2009). Type locality: Trinidad, Arima, Windblow Ridge, Simla House, 2000. Distr. - Colombia (Amazonas, Caldas, Córdoba, Valle del Cauca), Costa Rica (Cartago,
Higuito, Limón, Puntarenas, San José), Guatemala (Izabal), Nicaragua (Matagalpa), Panama (Chiriquí, Colón, Panamá Oeste), Suriname (Commewijne), Trinidad and Tobago (Trinidad), Venezuela (Carabobo, Miranda, Sucre). Holotype ơ', DEBU. Refs. - Marshall et al., 2009: 13 (fig., phyl.), 17 (key), 19 (distr. map), 30 (desc.), 33 (figs.), 34 (pl.), 45 (pl.), 67 (pl.); Marshall \& Buck, 2010: 844 (figs.); Rafael et al., 2011: 33 (fig., phyl.); Marshall, 2012: 475 (fig.); Rung, 2016: 559 (cat.).

carioca Prado, 1969: 20. Type locality: Brazil, Rio de Janeiro, Grajaú. Distr. - Brazil (Rio de Janeiro, São Paulo), Ecuador (Esmeraldas, Napo), Peru (Huánuco, Ucayali), Suriname (Sipaliwini), Venezuela (Monagas). Holotype ơ, FIOC. Refs. - Prado, 1969: 6 (key), 9 (fig.), 20 (desc.), 21 (figs.); Prado, 1975: 1 (cat.); Marshall et al., 2009: 17 (key), 19 (distr. map), 35 (redesc.), 36 (figs.), 45 (pl.), 74 (pl.); Rafael et al., 2011: 33 (fig., phyl.).

† craigi Grimaldi, 2009: 75 (in Marshall et al., 2009). Type locality: Dominican Republic [amber] (specific locality unknown). Distr. - Dominican Republic (Miocene). Holotype ơ, AMNH. Refs. - Marshall et al., 2009: 75 (desc.), 76 (figs.), 77 (fig.), 78 (pl.); Rafael et al., 2011: 33 (fig., phyl.).

cressoni Prado, 1969: 18. Typelocality:Brazil, Pará, Fazenda Velha. Distr. - Bolivia (La Paz), Brazil (Pará), Ecuador (Morona-Santiago, Napo), Peru (Cuzco, Huánuco, Lima), Suriname (Sipaliwini). Holotype ơ', FIOC. Refs. Cresson, 1914: 26 (male desc., fig.); Hennig, 1958: 542 (fig., as S. brunnea); Prado, 1969: 6 (key), 9 (fig.), 17 (figs.), 18 (desc.); Prado, 1975: 1 (cat.); Prado \& Papavero, 2002: 1 (cat.); Marshall et al., 2009: 17 (key), 19 (distr. map), 37 (redesc.), 38 (figs.), 45 (pl.); Rafael et al., 2011: 33 (fig., phyl.).

dactylopleura Marshall \& Buck, 2009: 40 (in Marshall et al., 2009). Type locality: Bolivia, La Paz, Arroyo Tuhiri, 5 km W Mapiri, 508 m, 15¹7.8'S, 68¹5.6’W. Distr. - Bolivia (La Paz), Ecuador (Napo, Orellana), Peru (Huánuco, Madre de Díos). Holotype o', CBFC. Refs. Marshall et al., 2009: 13 (fig., phyl.), 17 (key), 19 (distr. map), 40 (desc.), 42 (figs.), 58 (pl.), 67-68 (pls.); Rafael et al., 2011: 33 (fig., phyl.); Marshall, 2012: 475 (fig.).

fapeam Rafael, Câmara \& Holanda, 2011:27. Type locality: Brasil, Amazonas, Barcelos, Rio Padauari, Comunidade Ararinha, $00^{\circ} 30^{\prime} 18^{\prime \prime} \mathrm{N}, 64^{\circ} 03^{\prime} 30^{\prime \prime} \mathrm{W}$. Distr. - Brazil (Amazonas). Holotype on, INPA. Refs. - Rafael et al., 2011: 27 (desc.), 28 (figs.), 29 (key), 33 (phyl.).

figurata Marshall \& Buck, 2009: 43 (in Marshall et al., 2009). Type locality: Costa Rica, Puntarenas, Osa Península, $2.5 \mathrm{~km}$ S Rincón, $50 \mathrm{~m}, 08^{\circ} 42^{\prime} 01^{\prime \prime} \mathrm{N}$, $83^{\circ} 30^{\prime} 50^{\prime \prime}$ W. Distr. - Costa Rica (Higuito, San José, Puntarenas). Holotype ơ', INBC. Refs. - Marshall et al., 2009: 18 (key), 43 (desc.), 44 (figs.), 50 (distr. map), 58 (pl.), 74 (pl.); Rafael et al., 2011: 33 (phyl.).

lanei Prado, 1969: 6. Type locality: Brazil, São Paulo, Barueri. Distr. - Bolivia (La Paz), Brazil (São Paulo). Holotype ơ', MZUSP. Refs. - Prado, 1969: 6 (key, desc.), 7 (fig.), 9 (fig.), 11 (figs.); Griffiths, 1972: 166 (notes); Prado, 1975: 1 (cat.); Marshall et al., 2009: 17 (key), 19 (distr. map), 47 (redesc.); Mello \& Lamas, 2011: 188 (cat., type-material); Rafael et al., 2011: 33 (phyl.). 
lopesi Prado, 1969: 14. Type locality: Brazil, Bahia, Salvador. Distr. - Bolivia (La Paz), Brazil (Bahia, Pernambuco). Holotype ơ, FIOC. Refs. - Prado, 1969: 6 (key), 7 (fig.), 9 (fig.), 14 (desc.), 15 (figs.); Prado, 1975: 1 (cat.); Marshall et al., 2009: 17 (key), 45 (pl.), 48 (redesc.), 50 (distr. map); Mello \& Lamas, 2011: 189 (cat., type-material); Rafael et al., 2011: 33 (phyl.).

† miocenecus Grimaldi, 2009: 71 (in Marshall et al., 2009). Type locality: Dominican Republic [amber] (specific locality unknown). Distr. - Dominican Republic (Miocene). Holotype $\$$, AMNH. Refs. - Marshall et al., 2009: 71 (desc.), 73 (figs.), 77 (figs.), 78 (pl.); Rafael et al., 2011: 33 (phyl.).

nigrithorax Marshall \& Buck, 2009: 50 (in Marshall et al., 2009). Type locality: Ecuador, Departamento Orellana, near Yasuní Natl. Pk., Tiputini Biodiversity Station, 00³7'55"S, 7608'39"W, 220-250 m. Distr. - Ecuador (Orellana). Holotype o", USNM. Refs. - Marshall et al., 2009: 17 (key), 50 (fig.), 52 (figs.); Rafael et al., 2011: 33 (phyl.).

palenque Marshall \& Buck, 2009: 53 (in Marshall et al., 2009). Type locality: Ecuador, Província Pichincha, 47 km S Sto. Domingo, Río Palenque Biological Station, 250 m. Distr. - Colombia (Córdoba), Ecuador (Guayas, Pichincha). Holotype $\&$, DEBU. Refs. - Marshall et al., 2009: 17 (key), 19 (distr. map), 45 (pl.), 54 (figs.); Rafael et al., 2011: 33 (phyl.); Rung, 2016: 559 (cat.).

papaveroi Prado, 1969: 10. Type locality: Brazil, São Paulo, Barueri. Distr. - Brazil (Rio de Janeiro, São Paulo). Holotype ơ, MZUSP (Figs. 1-2). Refs. - Prado, 1969:6 (key), 7 (figs.), 9 (fig.), 10 (desc.), 13 (figs.), 23 (fig.); Prado, 1975: 1 (cat.); D. McAlpine, 1997: 191-192 (figs.), 194 (fig.); Marshall et al., 2009: 18 (key), 50 (distr. map), 55 (notes); Mello \& Lamas, 2011: 189 (cat., type-material); Rafael et al., 2011: 29 (key, redesc.), 30 (figs.), 33 (phyl.). Remarks: Syringogaster papaveroi was described as Stylogaster papaveroi, a lapsus calami (article 32.5, ICZN; Feijen, 1989). The species was correctly spelled on pages 1, 6, 7, 9, 12, 13 and 23 (Marshall et al., 2009), in Prado (1975) and so on.

plesioterga Marshall \& Buck, 2009: 56 (in Marshall et al., 2009). Type locality: Ecuador, Pto. Orellana, Río Tiputini, $00^{\circ} 38.2^{\prime} \mathrm{S}, 76^{\circ} 08.9^{\prime} \mathrm{W}$. Distr. - Ecuador (Napo, Orellana), Peru (Huánuco, Madre de Díos, Pasco). Holotype on, USNM. Refs. - Marshall et al., 2009: 18 (key), 50 (distr. map), 56 (desc.), 57 (figs.), 58 (pl.), 67-68 (pls.); Rafael et al., 2011: 33 (phyl.).

rufa Cresson, 1912: 393. Type locality: Costa Rica, Surubres River, Bonnefil farm, 800'. Distr. - Costa Rica (Alajuela, Heredia, Puntarenas), Panama (Panamá Oeste), Ecuador (Pichincha). Holotype \$, ANSP. Refs. - Cresson, 1912: pl. XIX (figs.), 393 (desc.); Papavero, 1964: 109-113 (notes); Roback, 1969: 555 (cat., type-material); Prado, 1969: 22 (notes); Griffiths, 1972: 290 (fig.); Prado, 1975: 1 (cat.); Buck et al., 2009: 150 (fig.); Marshall et al., 2009: 13 (phyl.), 18 (key), 34 (pl.), 50 (distr. map), 58 (pl.) 59 (redesc.), 60 (figs.), 67 (pl.), 68 (pl.); Rafael et al., 2011: 33 (phyl.).

sharkeyi Marshall \& Buck, 2009: 62 (in Marshall et al., 2009). Type locality: Peru, Departamento Huánuco,
Río Llullapichis, right tributary of Río Pachitea, Station "Panguana" (of H.-W. Koepcke), 09³6'53"S, 7455'57"W, 220 m. Distr. - Ecuador (Napo), Peru (Huánuco). Holotype o", DEBU. Refs. - Marshall et al., 2009: 17 (key), 50 (distr. map), 62 (desc.), 63 (figs.); Rafael et al., 2011: 33 (phyl.).

subnearctica Feijen, 1989: 119. Type locality: Mexico, San Luis Potosí, Tamazunchalo. Distr. - Costa Rica (Guanacaste, Higuito, Puntarenas), El Salvador (San Salvador), Guatemala (El Progreso), Mexico (Chiapas, San Luis Potosí, Tabasco, Veracruz). Holotype ơ, CAS. Refs. - Feijen, 1989: 119 (desc.), 120 (figs.), 121 (fig.); Marshall et al., 2009: 17 (key), 45 (pl.), 50 (distr. map), 64 (redesc.), 65 (figs.); Rafael et al., 2011: 33 (phyl.).

tenuipes Marshall \& Buck, 2009: 69 (in Marshall et al., 2009). Type locality: Bolivia, La Paz, Heath River Wildlife Centre, 12 $40^{\prime} \mathrm{S}, 68^{\circ} 42^{\prime} \mathrm{W}$. Distr. - Bolivia (La Paz), Ecuador (Napo), Peru (Madre de Díos). Holotype $\sigma^{\prime \prime}$, UASC. Refs. - Marshall et al., 2009: 13 (phyl.), 18 (key), 34 (pl.), 50 (distr. map), 69 (desc.), 70 (figs.); Rafael et al., 2011: 33 (phyl.).

\section{Groups and subgroups references}

brunnea-group. Refs. - Marshall et al., 2009: 11 (char., brunnea- and brunneina-subgroup), 12-13 (figs), 16 (key).

craigi-group. Refs. - Marshall et al., 2009: 11 (char.), 12 (figs.). figurata-group. Refs. - Marshall et al., 2009: 11 (char., figurata-subgroup), 12-13 (figs.).

rufa-group. Refs. - Marshall et al., 2009: 11 (char., laneiand rufa-subgroup), 12-13 (figs.), 18 (key); Rafael et al., 2011: 29 (key).

\section{Additional type material (paratypes)}

apiculata Marshall \& Buck; Paratypes: 10", DEBU; 10", MUSM; $10^{\circ}$, USNM.

atricalyx Marshall \& Buck; Paratypes: $20^{\circ} \sigma^{\pi} 39 \%, \mathrm{AMNH}$;

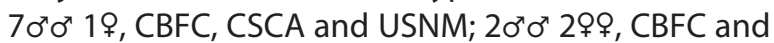

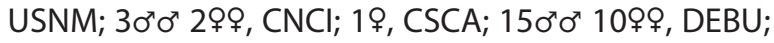
$50^{\prime \prime} \sigma^{\prime \prime} 4 \% \%$, DEBU and MUSM; $50^{\prime \prime} \sigma^{\prime \prime} 4 \%+$, DEBU and UASC; $60^{\prime \prime} \sigma^{\prime \prime} 1 \%$, ROME; 420'0' 22\%, USNM.

brachypecta Marshall \& Buck; Paratypes: $50^{\prime \prime} 0^{\prime \prime} 7$ 7\% DEBU and QCAZ; $1 \sigma^{\circ}$, LACM; 60" $\sigma^{\circ} 12 \% \%$, USNM.

brunnea Cresson; Paratype: $\sigma^{\prime \prime}$, ANSP.

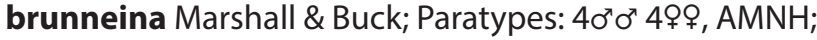

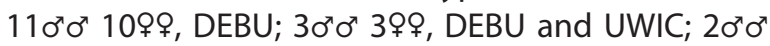

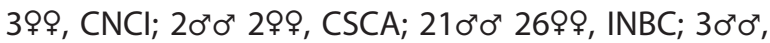

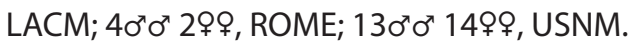

cressoni Prado; Paratype: $\sigma^{\prime \prime}$, FIOC.

dactylopleura Marshall \& Buck; Paratypes: $20^{7} 0^{7} 2$ 2क CBFC, CSCA and USNM; $19, \mathrm{CNCl}^{\prime} 60^{\prime \prime} 0^{\prime \prime} 2 \%$, DEBU; $50^{\top} 0^{\top} 4$ \% 9 , USNM.

fapeam Rafael, Câmara \& Holanda; Paratypes: $40^{\prime \prime} 0^{\top} 4$ 4 ${ }^{\circ}$, INPA; $10^{\prime} 19$, MZUSP.

figurata Marshall \& Buck; Paratypes: $30^{7} 0^{\pi} \mathrm{AMNH} ; 10^{\pi}, \mathrm{CNCl}$;

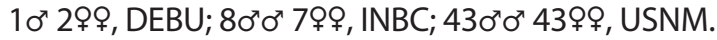

lanei Prado; Paratypes: $40^{\prime \top} \sigma^{\top} 3 \% \circ$, MZUSP. 
Iopesi Prado; Paratypes: 10 3\%क, FIOC; 10", MZUSP. Remarks: one paratype originally designated to be in FIOC is in MZUSP.

nigrithorax Marshall \& Buck; Paratype: 10", USNM.

palenque Marshall \& Buck; Paratypes: 1\%, DEBU; 10", AMNH; 19, USNM.

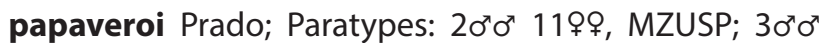
3 우, FIOC. Remarks: there are $20^{\prime \prime} \sigma^{\prime \prime} 11 \%$ p paratypes in MZUSP instead the $30^{\prime \prime} 0^{\prime \prime} 20 \%$ originally designated to be.

plesioterga Marshall \& Buck; Paratypes: 10" $\mathrm{CNCl} ; 10^{2}$, CSCA; $10^{\prime \prime}, \mathrm{ROME} ; 1 \%$, SMTD; 80"0 26\%, USNM.

rufa Cresson; Paratypes: $20^{\top} \sigma^{\prime \prime}$, ANSP.

sharkeyi Marshall \& Buck; Paratype: $19, \mathrm{CNCl}$.

tenuipes Marshall \& Buck; Paratypes: 1 으, $\mathrm{CNCl}$; $10^{\pi} 1$; DEBU; $10^{\prime} 3$ \% , $\mathrm{CNCl}$ and DEBU.

\section{ACKNOWLEDGMENTS}

We are very greatful to Dr. Carlos Lamas (MZUSP) by access to the type material of Syringogaster papaveroi Prado and to Camila Conti (MZUSP) by the photo of holotype of S. papaveroi. APC is grateful to Fundação de Apoio à Pesquisa do Estado da Paraíba (FAPESQ) and Conselho Nacional de Desenvolvimento Científico e Tecnológico (CNPq) for the research support (Proc. \#350052/2014-0).

\section{REFERENCES}

Brues, C.T. \& Melander, A.L. 1932. Classification of insects: a key to the known families of insects and other terrestrial arthropods. Bulletin of the Museum of Comparative Zoology at Harvard University, 73: 1-672. www. biodiversitylibrary.org/page/2800225\#page/5/mode/1up.

Brues, C.T.; Melander, A.L. \& Carpenter, F.M. 1954. Classification of insects: keys to the living and extinct families of insects, and to the living families of other terrestrial arthropods. Bulletin of the Museum of Comparative Zoology at Harvard University, 108: 1-917. www.biodiversitylibrary.org/ item/21151\#page/5/mode/1up.

Buck, M.; Woodley, N.E.; Borkent, A.; Wood, D.M.; Pape, T.; Vockeroth, J.R.; Michelsen, V. \& Marshall, S.A. 2009. Key to Diptera families - adults. In: Brown, B.V.; Borkent, A.; Cumming, J.M.; Wood, D.M.; Woodley, N.E. \& Zumbado, M.A. (Eds.). Manual of Central American Diptera. Ottawa, NRC Research Press. v. 1, p. 95-156.

Cresson, E.T. 1912. Descriptions of several new neotropical acalyptrate Diptera. Entomological News, 23(9): 389-396. www.biodiversitylibrary. org/item/84972\#page/7/mode/1up.

Cresson, E.T. 1914. The male of Syringogaster brunnea Cresson, from Peru (Dipt.). Entomological News, 25(1): 26. www.biodiversitylibrary.org/ item/20329\#page/62/mode/1up.

Curran, C.H. 1934. The families and genera of North American Diptera. New York, Ballou Press. 512p. +2 pls. DOI

Feijen, H.R. 1983. Systematics and phylogeny of Centrioncidae, a new afromontane family of Diptera (Schizophora). Zoologische Verhandelingen, 202: 3-137. www.repository.naturalis.nl/document/149145.

Feijen, H.R. 1989. Diopsidae. In: Griffiths, G.C.D. (Ed.). Flies of the Nearctic Region. Stuttgart, Schweizerbarth'sche. v. 9, 1-122.
Griffiths, G.C.D. 1972. The phylogenetic classification of the Diptera Schizophora with special reference to the structure of the male postabdomen. The Hague, W. Junk. 340p. (Series Entomologica 8). D0I

Hennig, W. 1958. Die Familien der Diptera Schizophora und ihre phylogenetischen Verwandtschaftsbeziehungen. Beiträge zur Entomologie, 8(5-6): 505-688. DOI

Hennig, W. 1965. Die Acalyptratae des Baltischen Bernsteins und ihre Bedeutung für die Erforschung der phylogenetischen Entwicklung dieser Dipteren-Gruppe. Stuttgarter Beiträge zur Naturkunde, 145: 1-215. www. biodiversitylibrary.org/page/33512937\#page/7/mode/1up

Hennig, W. 1973. 31. Diptera (Zweiflügler). In: Helmcke, J.-G.; Starck, D. \& Wermuth, H. (Eds.). Handbuch der Zoologie. Eine Naturgeschichte der Stämme des Tierreichs. IV. Band: Arthropoda - 2. Hälfte: Insecta. Zweite Auflage, 2, Teil: Spezielles. Berlin, W. De Gruyter. p. iii + 1-337.

Marshall, S.A. 2012. Flies: the natural history and diversity of Diptera. Richmond Hill, Firefly Press Ltd. p. 1-616.

Marshall, S.A. \& Buck, M. 2010. Syringogastridae (Syringogastrid flies). In: Brown, B.V.; Borkent, A.; Cumming, J.M.; Wood, D.M.; Woodley, N.E. \& Zumbado, M.A. (Eds.). Manual of Central American Diptera. Ottawa, NRC Research Press. v. 2, p. 843-846.

Marshall, S.A.; Buck, M.; Skevington, J.H. \& Grimaldi, D. 2009. A revision of the family Syringogastridae (Diptera: Diopsoidea). Zootaxa, 1996: 1-80.

McAlpine, D.K. 1997. Gobryidae, a new family of acalyptrate flies (Diptera: Diopsoidea), and a discussion of relationships of the diopsoid families. Records of the Australian Museum, 49: 167-194. D0I

McAlpine, D.K. 2011. Observations on antennal morphology in Diptera, with particular reference to the articular surfaces between segments 2 and 3 in the Cyclorrhapha. Records of the Australian Museum, 63: 113-166. D0I

McAlpine, J.F. 1989. Phylogeny and classification of the Muscomorpha. In: McAlpine J.F. \& Wood, D.M. (Eds.). Manual of Nearctic Diptera. Ottawa, Research Branch, Agriculture Canada. v. 3, p. 1397-1518. (Monograph 32). http://publications.gc.ca/site/eng/9.817751/publication.html.

Meier, R. \& Hilger, S. 2000. On the egg morphology and phylogenetic relationships of Diopsidae (Diptera: Schizophora). Journal of Zoological Systematics and Evolutionary Research, 38(1): 1-36. D0

Mello, R.L. \& Lamas, C.J.E. 2011. A catalogue of types of Conopoidea, Diopsoidea, Nerioidea and Tephritoidea (Diptera, Schizophora) in the collection of the Museu de Zoologia da Universidade de São Paulo. Revista Brasileira de Entomologia, 55(2): 187-205. www.scielo.br/pdf/rbent/ v55n2/A0P1811.pdf.

Papavero, N. 1964. Notes on the myrmecomimicry of Syringogaster rufa Cresson (Diptera, Acalyptratae, Megamerinidae). Papéis Avulsos de Zoologia, 16(12): 110-113.

Prado, Â.P. do. 1969. Syringogastridae, uma nova família de dípteros Acalyptratae, com a descrição de seis espécies novas do gênero Syringogaster Cresson. Studia Entomologica, 12(14): 1-32.

Prado, Â.P. do. 1975. Family Syringogastridae. In: Papavero, N. (Ed.). A Catalogue of Diptera of the Americas South of the United States. São Paulo, Departamento de Zoologia, Secretaria da Agricultura. n. 51, p. 1-2. http://biodiversitylibrary.org/page/50660667.

Prado, A.P. do \& Papavero, N. 2002. Insecta - Diptera - Syringogastridae. Fauna da Amazônia Brasileira, 4: 1.

Rafael, J.A.; Câmara, J.T. \& Holanda, M.J.A. 2011. A new species of Syringogastridae (Diptera, Acalyptratae) from the Amazon Basin and new records for Brazil. Zootaxa, 3014: 26-34.

Roback, S.S. 1969. The genera, subgenera and species described by E.T. Cresson, Jr. 1906-1949. Transactions of the American Entomological Society, 95:517-569.

Rung, A. 2016. Family Syringogastridae. Zootaxa, 4122(1): 558-560. 